\title{
Antibacterial Activity and Cytocompatibility of PLGA/CuO Hybrid Nanofiber Scaffolds Prepared by Electrospinning
}

\author{
Adnan Haider, ${ }^{1}$ Sanghwa Kwak, ${ }^{1}$ Kailash Chandra Gupta, ${ }^{1,2}$ and Inn-Kyu Kang ${ }^{1}$ \\ ${ }^{1}$ Department of Polymer Science and Engineering, School of Applied Chemical Engineering, Kyungpook National University, \\ Daegu 702-701, Republic of Korea \\ ${ }^{2}$ Polymer Research Laboratory, Department of Chemistry, IIT, Roorkee 247667, India
}

Correspondence should be addressed to Inn-Kyu Kang; ikkang@knu.ac.kr

Received 8 December 2014; Revised 21 February 2015; Accepted 5 March 2015

Academic Editor: Tamer Uyar

Copyright (c) 2015 Adnan Haider et al. This is an open access article distributed under the Creative Commons Attribution License, which permits unrestricted use, distribution, and reproduction in any medium, provided the original work is properly cited.

\begin{abstract}
The PLGA/CuO hybrid nanofibers scaffolds were prepared via electrospinning technique. The presence of CuO in the PLGA scaffolds was confirmed by transmission electron microscope (TEM) and X-ray photoelectron spectroscopy (XPS). The scaffolds were subjected to various antibacterial and cytobiocompatibility tests. The results not only showed excellent adhesion, proliferation, and viability (live/dead staining) for fibroblastic cells but also revealed that PLGA/CuO hybrid nanofiber scaffolds inhibited both Gram-positive and Gram-negative bacterial growth. The mechanism of the antibacterial activity was concluded to be based on the $\mathrm{CuO}$ nanoparticles and $\mathrm{Cu}^{++}$ions release. It is, therefore, evaluated that PLGA/CuO hybrid nanofiber scaffolds can be a useful candidate for wound dressing.
\end{abstract}

\section{Introduction}

The emergence of infectious diseases due to microbes in general and the antibiotic-resistant bacterial strains (Grampositive and Gram-negative) in particular poses a serious threat to public health worldwide [1]. Both Gram-positive and Gram-negative bacterial strains are considered a major threat to animal's life $[1,2]$. In the last century, antibiotics were used as the only source for controlling and/or curing the infections caused by microbes. The current advances in the field of nanotechnology, especially, having the facility and ability to prepare metal or metal oxide nanoparticles of specific size and shape, have enabled human beings to invent new techniques for the preparation of antimicrobial agents against the microbes [2]. Microbial activity of the nanoparticles in part depends on their size, stability, and concentration of the nanoparticles in the growth medium. These properties of nanoparticles can easily be altered. Nanoparticles have therefore received great attention in the medicine field [3]. Considering the unique properties, nanosized inorganic particles such as metal oxides of zinc $(\mathrm{Zn})$, magnesium $(\mathrm{Mg})$, silver $(\mathrm{Ag})$, and copper $(\mathrm{Cu})$ are being generated for the ultimate use in biomedical nanotechnology
$[4,5]$. Antimicrobial activity of nanoparticles has largely been studied with human pathogenic bacteria such as Escherichia coli and Staphylococcus aureus [4]. The microbial cells and their colonies showed vulnerability to metals nanoparticles such as $\mathrm{Zn}, \mathrm{Ag}, \mathrm{Cu}$, and $\mathrm{Mg}[3,5,6]$. The bacterial population growth is inhibited by specific interaction of these metal nanoparticles with bacterial strains $[1,6]$. Among these metal nanoparticles, $\mathrm{Cu}$ and copper oxide $(\mathrm{CuO})$ nanoparticles have attracted considerable attention because $\mathrm{Cu}$ is easily available and one of the most widely used metals in the modern research world [7]. Since researchers have well documented the synthesis and optical, catalytic, and electrical properties and medicinal properties of $\mathrm{CuO}$ nanoparticles $[8,9]$, this study has chosen compositing $\mathrm{CuO}$ with the polymer for the ease of processability and developing a hybrid antibacterial scaffold [10].

Polymeric scaffolds play a vital role in tissue regeneration [11]. They are intended to provide favorable environment to the cells that ultimately help in stimulating their growth and normal cellular functions. Polymeric scaffolds help in the movement of various growth factors, metabolites, and soluble drugs that help in stimulating cell growth and help in tissue regeneration. Poly(lactide-co-glycolide) (PLGA) has 
been the most frequently used polymeric materials among the various polymeric materials used for the preparation of electrospun nanofiber scaffolds [11]. PLGA has been approved by the Food and Drug Administration (FDA) authority due to its biocompatible and biodegradable nature $[12,13]$. Furthermore PLGA has been extensively studied in the field of drug delivery and tissue regeneration. Apart from the biocompatible nature, PLGA offers different degradation time by tailoring the monomer ratio of lactide and glycolide [12].

Till now various techniques have been introduced for the preparation of various polymeric scaffolds including gas forming, freeze-drying, phase separation, and solvent casting. Among the so far used techniques, electrospinning is a simple, versatile, and cost effective technique used for generating fibers with size ranging from submicron $(\mu \mathrm{m})$ to nanometer $(\mathrm{nm})$ scale diameter $[13,14]$. Electrospinning is used for producing multifunctional nanofiber from both natural and artificial polymers, polymer blends, and composite [15]. Along with high surface area, inter- and intrafibrous pores, electrospun nanofiber scaffolds have a strong adhesive force, good air filtration, high adhesion barrier activity, and heat resistance [15-17]. These properties make these nanofiber scaffolds quite similar in shape to human skin. The scope of electrospun nanofiber has been explored specifically in biomedical area for various reasons such as biofilms, wound dressing materials, hemostatic materials, artificial blood vessels, drug and gene delivery, antimicrobial agent, and tissue regeneration scaffolds $[3,18]$.

Keeping these antecedents in mind, the purpose of this study was to test and determine the antibacterial activity of PLGA/CuO hybrid nanofiber scaffolds on various bacterial strains. Furthermore, we aimed to study the interaction of fibroblast (skin cells) with PLGA/CuO hybrid nanofiber scaffolds so that it can be used as an internal and external wound dressing agent. This kind of study will pave new ways in designing the future wound dressing materials, which can behave as an antibacterial agent without compromising its cytocompatibility.

\section{Experimental}

2.1. Materials and Methods. Poly(lactide-co-glycolide) (PLGA), copper (Cu), dimethylformamide (DMF), and tetrahydrofuran (THF) were purchased from Sigma Aldrich. Fibroblastic cell line (NIH3T3) was obtained from Korea Cell Bank. MTT assay kit was purchased from Gibco, Invitrogen, USA, whereas Dulbecco's modified eagle's medium (DMEM), fetal bovine serum (FBS), and penicillin G-streptomycin were acquired from Gibco, Japan. Escherichia coli (KCCM 12119) and staphylococcus aureus (KCCM 12256) were obtained from Korean Culture Center of Microorganisms (KCCM). All the reagents and chemicals in this study were used as received.

2.2. Synthesis of Copper Oxide (CuO) Nanoparticles. For the synthesis of $\mathrm{CuO}$ nanoparticles, $3 \mathrm{mg}$ of $\mathrm{Cu}$ powder was added to $30 \mathrm{~mL}$ of distilled water in a glass vial and was sonicated for about 25 minutes in a sonicator. The reaction mixture was transferred into a glass bottle and sealed under ordinary conditions by wrapping the bottle cap with Teflon foil. The sealed glass bottle containing reaction solution was autoclaved at $140^{\circ} \mathrm{C}$ for 24 hours. After 24 hours the solution was allowed to cool at room temperature and then centrifuged to retrieve $\mathrm{CuO}$ nanoparticles. The nanoparticles were washed repeatedly with double distilled water, freezedried, and stored [19].

2.3. Solution Preparation and Fabrication of Nanofiber Scaffolds. Polymer solutions at the concentration of 5 to $20 \mathrm{wt} \%$ were prepared by dissolving PLGA in a binary solvent of THF and DMF in 3:1 ratio. The solution was stirred overnight at room temperature until complete dissolution. The prepared solution was then subjected to electrospinning process. The typical electrospinning apparatus and the basic concept of the fiber formation are summarized in Figure 1. Briefly, the solution was transferred to a $10 \mathrm{~mL}$ glass syringe fitted with a needle with an inner diameter of $0.9 \mathrm{~mm}$. The electrospinning experiment was based on our previously reported study [13]. The optimized electrospinning conditions used in the present study were tip to collector distance $20 \mathrm{~cm}$, applied voltage $20 \mathrm{kV}$, and a solution flow rate $1 \mathrm{~mL} / \mathrm{h}$ which was maintained throughout the electrospinning process. The electrospun nanofiber scaffolds were collected onto the aluminium foil wrapped over the metallic collector. After the completion of the process, the electrospun nanofiber scaffolds were removed from the metallic collector along with the aluminium foil. The electrospun nanofiber scaffold was vacuum dried overnight at $40^{\circ} \mathrm{C}$ to remove the solvent. The same procedure was adapted for the preparation of the electrospun PLGA/CuO hybrid nanofiber scaffolds containing $0.5 \mathrm{wt} \%$ of $\mathrm{CuO}$ nanoparticles.

2.4. Characterization of the Scaffolds. The viscosity of the PLGA polymer solutions in the binary solvent (THF: DMF = $3: 1$ ratio) was measured at room temperature using a viscometer (Brookfield Viscometer DV-II Pro) with spindle number 6 at 100 revolutions per minute (rpm). The morphology of the PLGA/CuO hybrid nanofiber scaffolds was evaluated by field emission scanning electron microscopy (FESEM, 400 Hitachi, Tokyo, Japan) and transmission electron microscope (BioTEM, Hitachi, Tokyo, Japan). The particle size was analyzed via dynamic light scattering (DLS). The qualitative and quantitative analysis of $\mathrm{CuO}, \mathrm{PLGA} / \mathrm{CuO}$, and PLGA nanofiber scaffolds were carried out by X-ray photoelectron spectroscopy (XPS, ESCA LAB VIG microtech, Mt 500/1, etc., East Grinstead, UK) equipped with $\mathrm{Mg} \mathrm{K \alpha}$ radiation at $1,253.6 \mathrm{eV}$ and a $150-\mathrm{W}$ power mode at the anode. A survey scan spectrum was taken and the surface elemental compositions relative to the carbon were calculated from the peak heights taking into account the atomic sensitivity.

2.5. Antibacterial Activity. The antibacterial activities of pristine PLGA and PLGA/CuO nanofiber scaffolds were investigated against model microbial species including Escherichia coli (Gram-negative) and Staphylococcus aureus, 


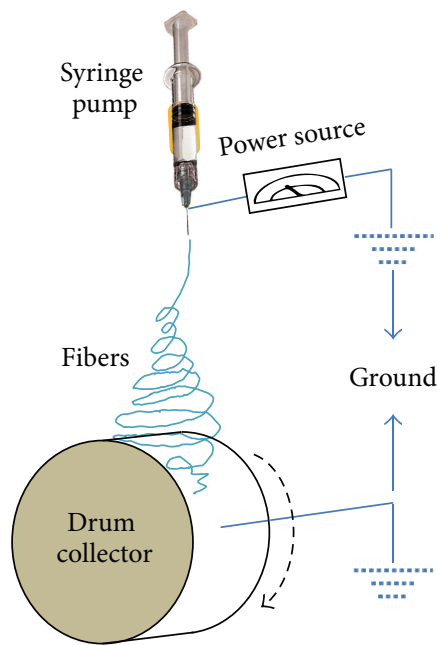

(a)

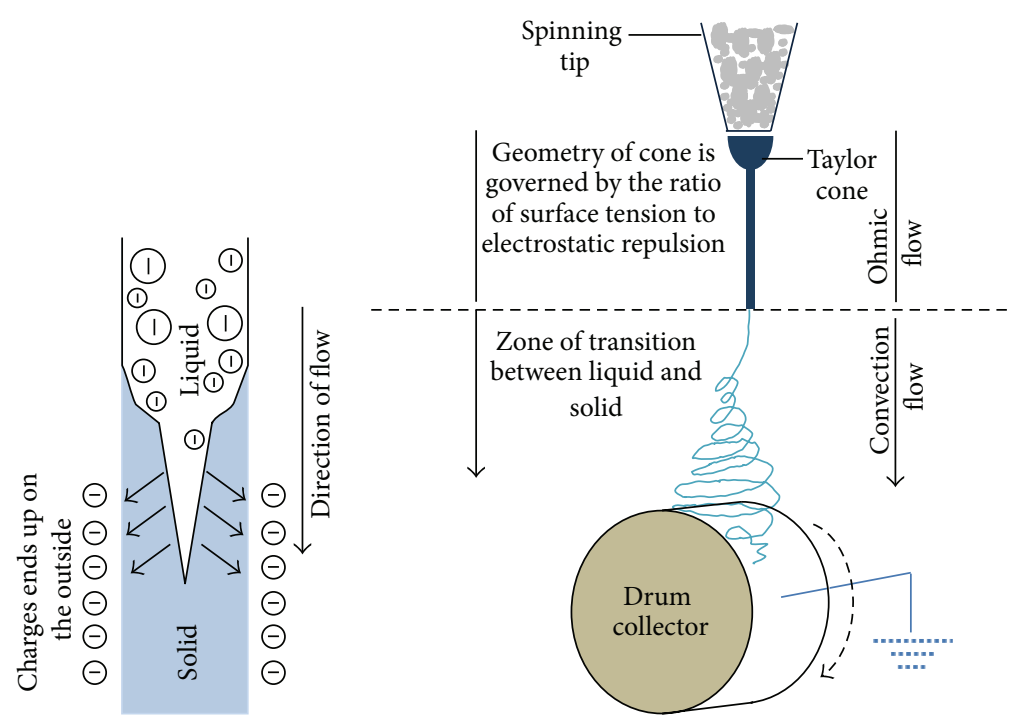

(b)

(c)

FIGURE 1: The schematic of the basic concept of electrospinning: electrospinning apparatus (a), accumulation of charges on the needle and consequently on the liquid (b), and Taylor cone and basic schematic for nanofiber preparation (c).

S. aureus (Gram-positive), through two well-known methods described below.

2.5.1. Agar Disc Diffusion Method. In this method, the antibacterial activities of the pristine PLGA and PLGA-CuO hybrid nanofiber scaffolds were measured by disc diffusion method [20]. Microbial species, E. coli (KCCM 12119), was grown on nutrient agar (DIFCO 0001) containing $3 \mathrm{~g} / \mathrm{L}$ beef extract, $5 \mathrm{~g} / \mathrm{L}$ peptones, and $15 \mathrm{~g} / \mathrm{L}$ agar in distilled water, whereas S. aureus (KCCM 12256) was grown on trypticase soy agar (BBL 211768) containing $17 \mathrm{~g} / \mathrm{L}$ pancreatic digest of casein, $3 \mathrm{~g} / \mathrm{L}$ pancreatic digest of soybean meal, and $15 \mathrm{~g} / \mathrm{L}$ agar in distilled water. The $\mathrm{pH}$ of the agar media for both microbial species was maintained at 7.0 [20]. Disc shape samples of PLGA and PLGA/CuO hybrid nanofiber scaffolds with $1 \times 1 \mathrm{~cm}^{2}$ dimension were prepared and subjected to the inhibition zone test. The samples were sterilized with UV for 2 hours and subsequently placed on E. coli and S. aureus culture plates. The plates were incubated for 24 hours at $37^{\circ} \mathrm{C}$. The relative antibacterial effect was found by measuring the clear zones of inhibition formed around the discs [21].

2.5.2. Optical Density Method. In this method, pristine PLGA and PLGA-CuO hybrid nanofiber scaffolds were sliced into small pieces and sterilized at $121^{\circ} \mathrm{C}$ for 15 minutes. Next, $50 \mathrm{~mL}$ of growth medium for both bacterial species was taken in $100 \mathrm{~mL}$ flasks, followed by addition of $0.005 \mathrm{~g} / \mathrm{mL}$ finely sliced solid PLGA and PLGA-CuO hybrid nanofiber scaffolds. The tubes were then seeded with $1 \mathrm{~mL}$ fresh culture of bacterial strains and incubated in a shaking incubator at $37^{\circ} \mathrm{C}$ and for 24 hours. The turbidity of the media was observed at various time intervals at $610 \mathrm{~nm}$ using a UV spectrophotometer (T60U, China) [20, 22].
2.6. Cell Adhesion Study. To examine the response of the fibroblastic cells to the pristine PLGA and PLGA/CuO hybrid nanofiber scaffolds, small circular samples of $1 \times 1 \mathrm{~cm}^{2}$ dimension of both scaffolds were prepared and used. Briefly, the circular samples of the PLGA and PLGA/CuO hybrid nanofiber scaffolds were fitted in a 24-well culture dish and subsequently immersed in a DMEM medium containing $10 \%$ FBS and $1 \%$ penicillin G-streptomycin. One milliliter of a NIH3T3 cell solution $\left(5 \times 10^{5}\right.$ cells $\left./ \mathrm{mL}\right)$ was seeded on the electrospun pristine and PLGA/CuO hybrid nanofiber scaffolds and incubated in a humidified atmosphere $\left(5 \% \mathrm{CO}_{2}\right.$ and at $37^{\circ} \mathrm{C}$ ) for 1 and 3 days in order to determine the cell adhesion on the nanofiber scaffolds. After incubation, the supernatant was removed, washed twice with PBS, and fixed with an aqueous $2.5 \%$ glutardialdehyde solution for 20 minutes. The sample sheet was then dehydrated, dried in a critical point drier, and stored for characterization [3].

2.7. Cell Proliferation and Viability Study. 3-(4, 5-Dimethylazol-2-yl)-2,5-diphenyl-2H-tetrazolium bromide (MTT) assay was used to determine the proliferation of fibroblastic cells on the PLGA and PLGA/CuO hybrid nanofiber scaffolds. Briefly, NIH3T3 cells were seeded at a concentration of $3 \times 10^{4}$ cells/mL onto pristine PLGA and PLGA/CuO hybrid nanofiber scaffolds, which were fitted in a 24-well plate, and cell proliferation was monitored after 1 and 3 days of incubation. A MTT solution $(50 \mu \mathrm{L}, 5 \mathrm{mg} / \mathrm{mL}$ in PBS) was added to each well and incubated in a humidified atmosphere containing $5 \% \mathrm{CO}_{2}$ at $37^{\circ} \mathrm{C}$ for 4 hours. After removing the medium, the converted dye was dissolved in acidic isopropanol (0.04 $\mathrm{N} \mathrm{HCl}$-isopropanol) and kept in the dark at room temperature for 30 minutes. From each sample, $100 \mu \mathrm{L}$ medium was taken, transferred to a 96-well plate, and 


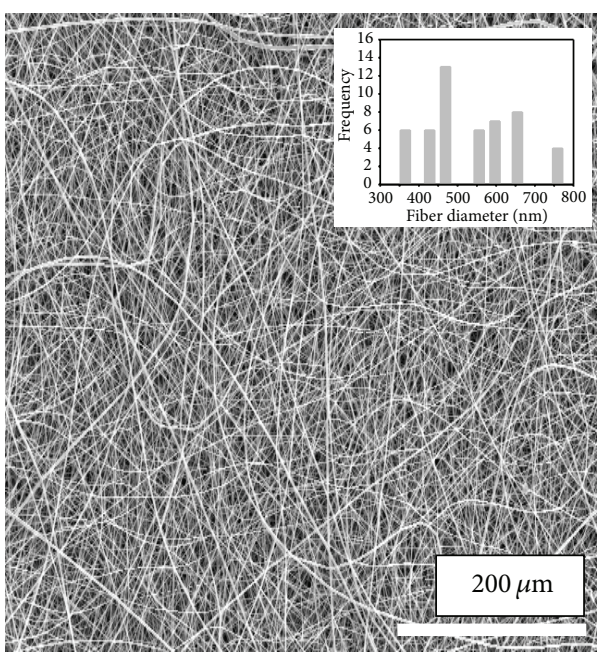

(a)

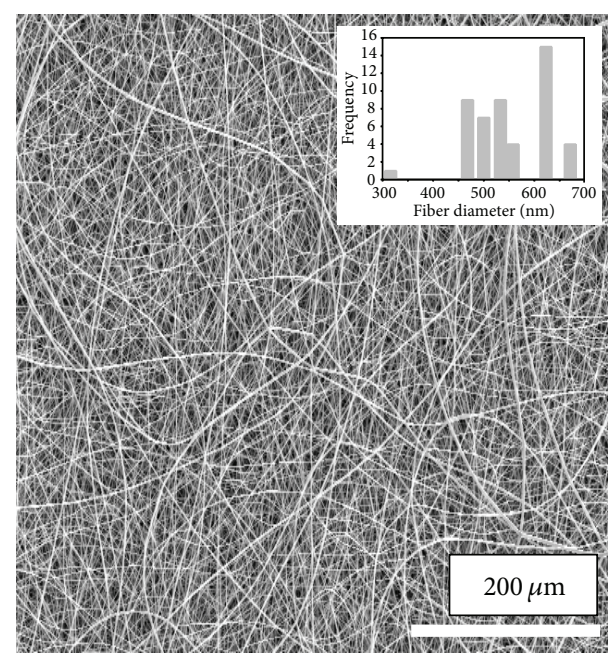

(b)

FIGURE 2: FE-SEM images of PLGA (a) and PLGA/CuO hybrid nanofiber scaffolds (b).

subjected to ultraviolet measurements for the converted dye at a wavelength of $570 \mathrm{~nm}$ on a kinetic microplate reader $(\mathrm{EL} \times 800$, Bio-T Instruments, Inc., Highland Park, USA).

A standard live/dead assay was conducted for the evaluation of NIH3T3 cell viability after culturing NIH3T3 cells on pristine PLGA and PLGA/CuO hybrid nanofiber scaffolds. The cell viability experiment was performed in accordance with the previous articles $[3,23]$.

2.8. $\mathrm{Cu}^{++}$Ion Release. The $\mathrm{Cu}^{++}$ions release experiment was carried out to find the ionization potential of $\mathrm{CuO}$ from the PLGA/CuO hybrid nanofiber scaffolds. The amount of $\mathrm{Cu}^{++}$ ions released from the sample was determined by immersing the PLGA/CuO hybrid nanofiber scaffolds $\left(4 \times 4 \mathrm{~cm}^{2}, 0.2 \mathrm{~g}\right)$ into $10 \mathrm{~mL}$ distilled water for different time periods. The amount of $\mathrm{Cu}^{++}$ions in the double distilled water was determined by inductively coupled plasma spectrophotometer (ICP, Thermo Jarrell Ash IRIS-AP) [3].

\section{Results and Discussion}

3.1. Morphology of Nanofiber and $\mathrm{CuO}$. Figure 2 depicts the FE-SEM images of the electrospun PLGA and PLGA/CuO hybrid nanofiber scaffolds. It is evident from the FE-SEM images of PLGA nanofiber (Figure 2(a)) and PLGA/CuO hybrid nanofiber scaffolds (Figure 2(b)) that after implementing optimized electrospinning parameters the electrospun nanofiber was smooth and uniform. The average diameters calculated from the histograms of the electrospun pristine PLGA (inset of (Figure 2(a)) and PLGA/CuO (inset of (Figure 2(b)) hybrid nanofiber scaffolds were 548 and $553 \mathrm{~nm}$, respectively. This small change in the average diameter of the fibers shows that the addition of $\mathrm{CuO}$ to PLGA did not affect the average diameter of the fibers to a great extent.

TEM images of the $\mathrm{CuO}$ nanoparticles and PLGA/CuO hybrid nanofiber are depicted in Figures 3(a) and 3(b).
Images show that $\mathrm{CuO}$ nanoparticles (Figure 3(a)) were successfully incorporated into the PLGA nanofiber (Figure 3(b)). The size distribution of $\mathrm{CuO}$ nanoparticles calculated from TEM (Figure 3(a)) was ranging from 40 to $100 \mathrm{~nm}$, whereas the relative size distribution obtained from DLS (inset of Figure 3(a)) was in the range of $60-128 \mathrm{~nm}$ with the maximum distribution peak observed at $95 \mathrm{~nm}$. Hence it was concluded that the size of the $\mathrm{CuO}$ ranged from 40 to $128 \mathrm{~nm}$.

3.2. XPS Study. ESCA survey scan spectra were used to confirm the presence of $\mathrm{CuO}$ nanoparticles in the matrix of PLGA electrospun nanofiber. $\mathrm{Cu}$ peak is the marker of choice for confirming the presence of $\mathrm{CuO}$ nanoparticles in the PLGA/CuO hybrid nanofiber scaffolds. The survey scan spectra of pristine PLGA nanofiber scaffolds show typical peaks of carbon C1s at $284.6 \mathrm{eV}$ and oxygen $\mathrm{O} 1 \mathrm{~s}$ at $536.1 \mathrm{eV}$ (Figure 4(a)). Similarly, two characteristic signals of $\mathrm{Cu}$ at $932.4 \mathrm{eV}$ and oxygen $\mathrm{O} 1 \mathrm{~s}$ at $536.1 \mathrm{eV}$ were detected in the survey scan spectra of pure $\mathrm{CuO}$ nanoparticles (Figure 4(c)). The appearance of the characteristic peaks of $\mathrm{Cu}$, carbon, and oxygen at $932.4 \mathrm{eV}, 284.6 \mathrm{eV}$, and $536.1 \mathrm{eV}$, respectively, in the survey scan spectra of PLGA/CuO hybrid nanofiber scaffolds confirmed the presence of $\mathrm{CuO}$ nanoparticles in the PLGA polymer matrix (Figure 4(b)). Besides the qualitative analysis of the survey scans from the peak positions, quantitative determination of the major elements was performed by analyzing the peak intensity and summarized in Table 1. The analysis of the data showed that after the successful incorporation of $\mathrm{CuO}$ nanoparticles into the PLGA/CuO hybrid nanofiber scaffold, a change in the \% weights of carbon (62.10), oxygen (47.53), and $\mathrm{CuO}(0.40)$ for PLGA/CuO hybrid nanofiber scaffolds was observed as compared to the pristine $\mathrm{CuO}$ nanoparticles and PLGA nanofiber. Therefore, it was confirmed from the ESCA that $\mathrm{CuO}$ nanoparticles were successfully incorporated into PLGA during electrospinning to prepare PLGA/CuO hybrid nanofiber scaffold. 


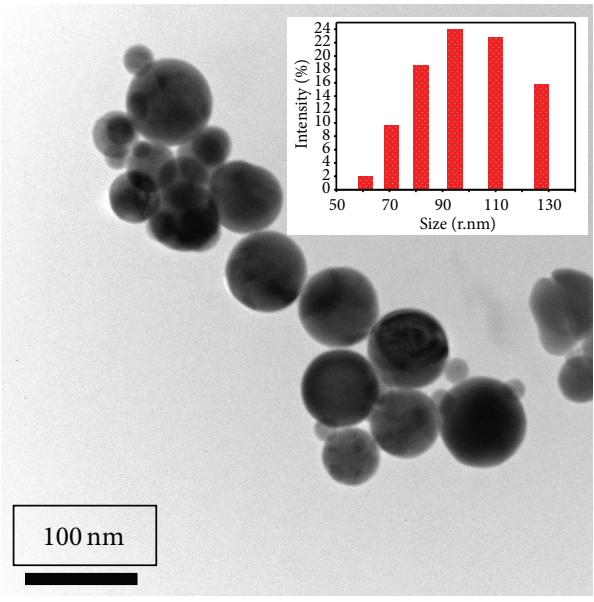

(a)

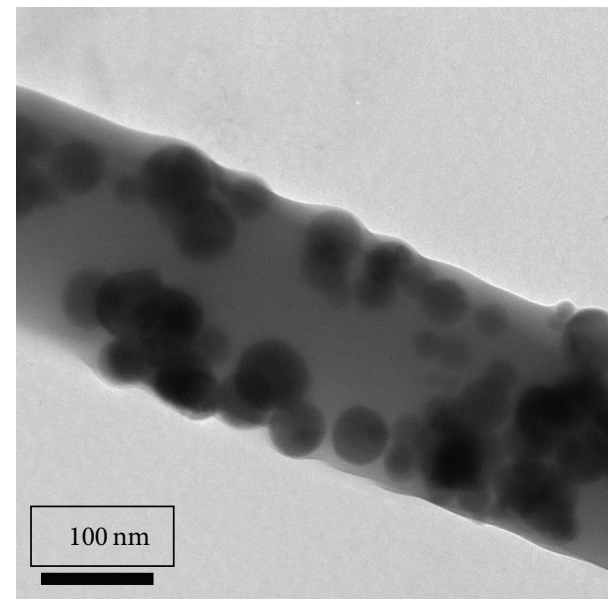

(b)

FIgUre 3: TEM images of the CuO nanoparticles (a) and PLGA/CuO electrospun hybrid nanofiber (b).

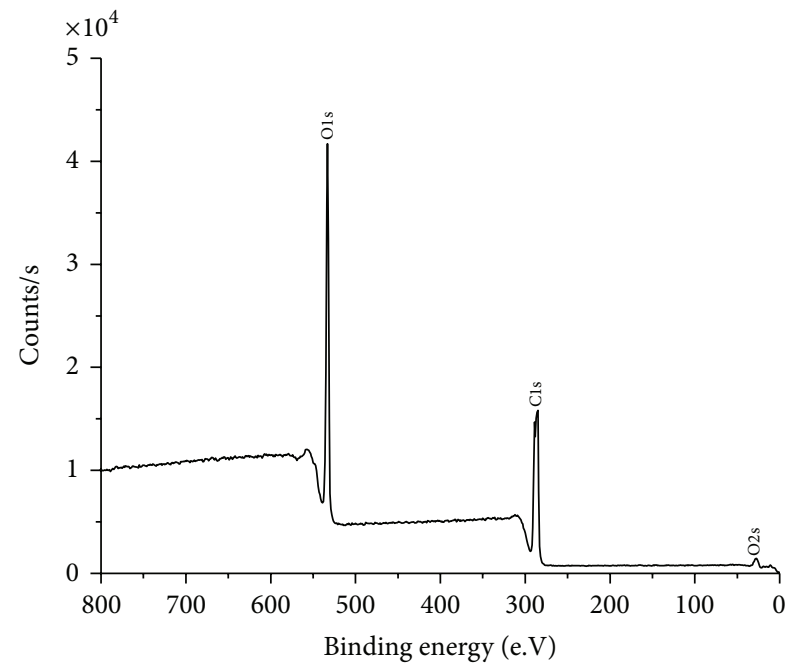

(a)

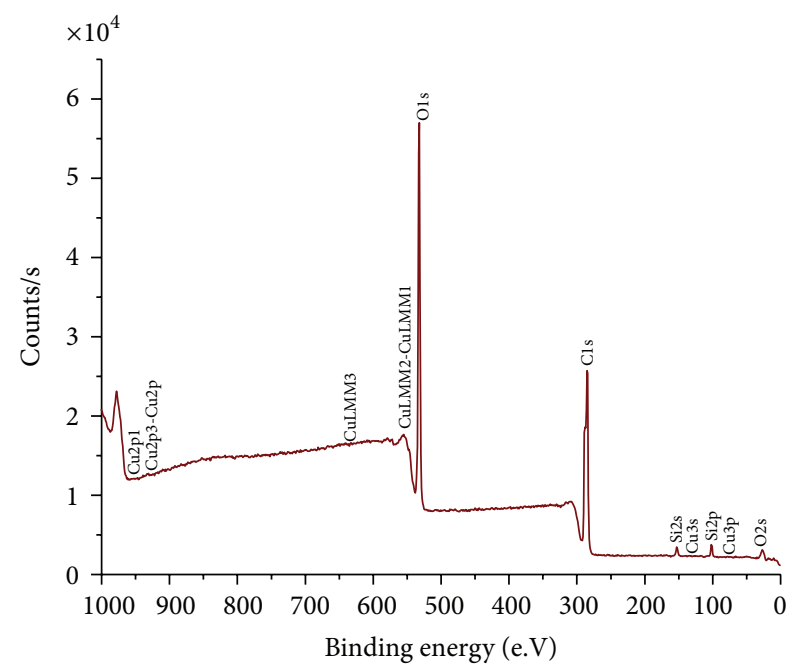

(b)

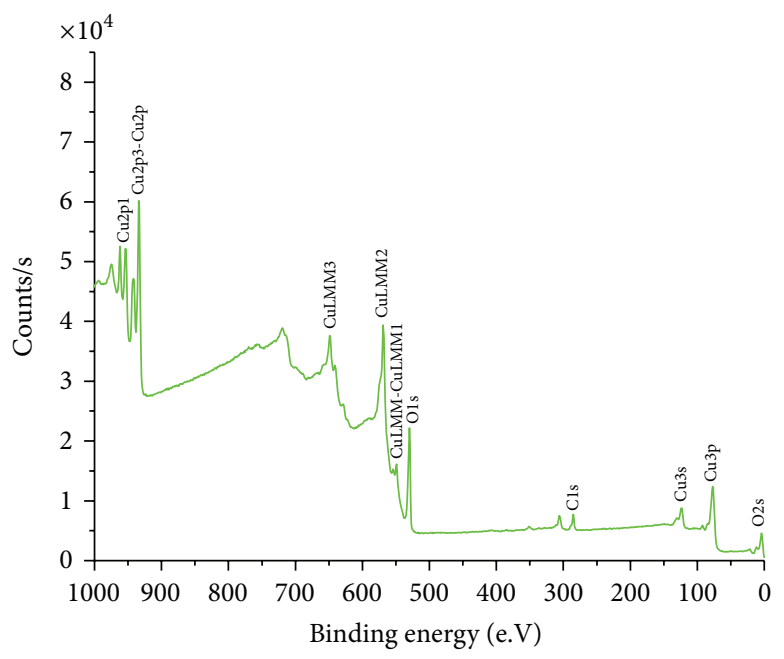

(c)

FIGURE 4: ESCA survey scan spectra of pristine PLGA (a), CuO nanoparticles (b), and (c) PLGA/CuO hybrid nanofiber scaffold. 


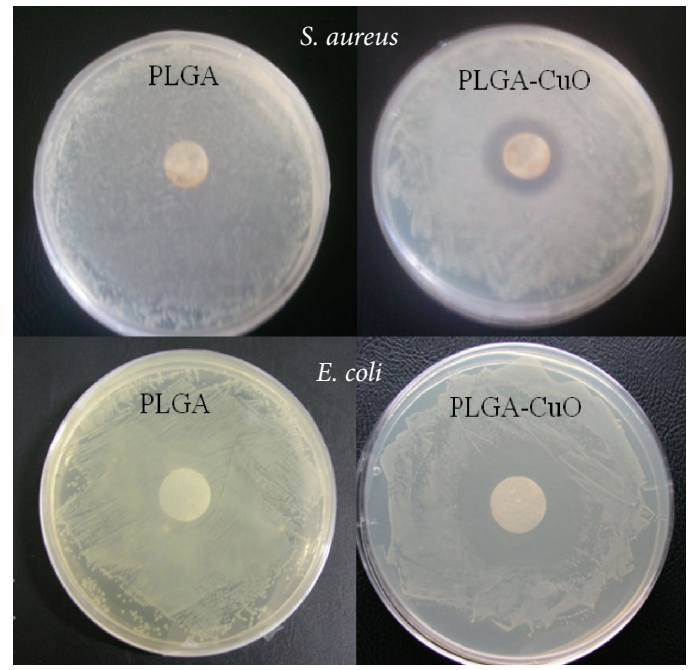

(a)

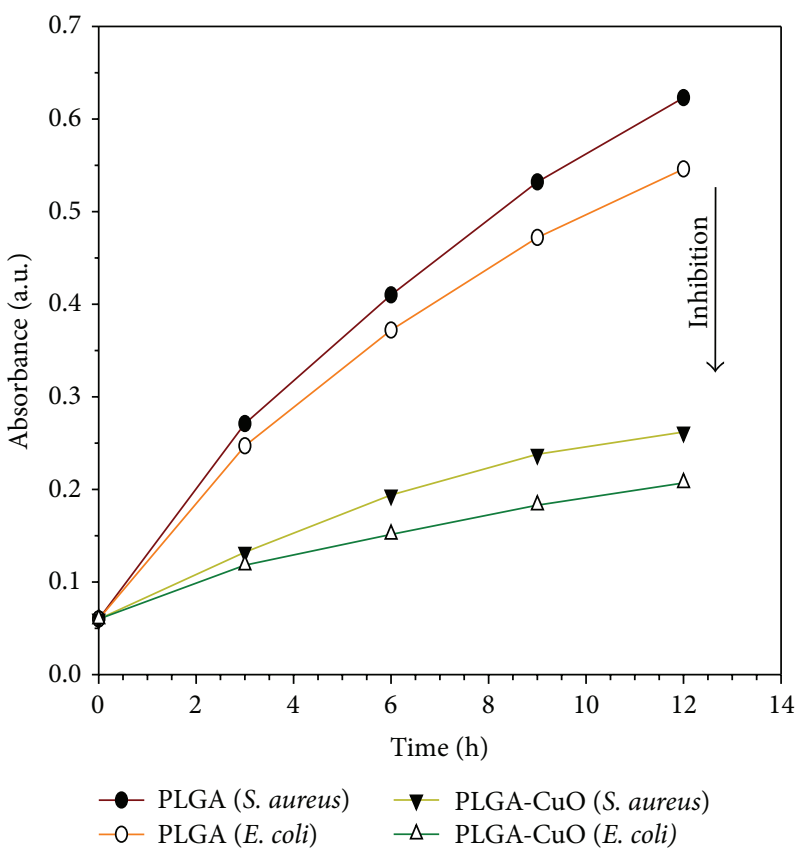

(b)

Figure 5: Inhibition zones of the PLGA and PLGA/CuO nanofiber scaffolds against E. coli and S. aureus determined by disc diffusion method (a) and antibacterial activities of PLGA and PLGA/CuO nanofiber scaffolds against $E$. coli and $S$. aureus determined by optical density method (b).

TABLE 1: Chemical composition of the CuO, PLGA, and PLGA/CuO composite nanofiber scaffolds calculated from ESCA survey scan spectra.

\begin{tabular}{lccc}
\hline Substrates & \multicolumn{3}{c}{ Atomic percent (\%) } \\
& $\mathrm{C}$ & $\mathrm{O}$ & $\mathrm{Cu}$ \\
\hline PLGA & 64.61 & 35.39 & \\
$\mathrm{CuO}$ & 6.23 & 43.40 & 49.70 \\
PLGA/CuO & 62.10 & 35.53 & 0.40 \\
\hline
\end{tabular}

3.3. Antibacterial Properties of PLGA and PLGA-CuO Hybrid Nanofiber Scaffolds. Lack of antibacterial properties is one of the serious obstacles limiting biomedical applications of biopolymers including PLGA. As being used in wound healing and tissue regeneration, the PLGA scaffolds should keep the ability of protection against pathogenic species [12, 13]. The current PLGA/CuO hybrid nanofiber scaffolds were prepared with the aim of introducing antibacterial activities into the PLGA electrospun nanofiber so as to enhance their applicability in medical field. Figures 5(a) and 5(b) illustrate the comparative results of the antibacterial activities of pristine PLGA and PLGA/CuO nanofiber scaffolds. The results obtained from disc diffusion method against $E$. coli and $S$. aureus indicated that the pristine PLGA nanofiber scaffold did not produce any zone of inhibition against both species (E. coli and S. aureus (Figure 5(a)). Thus the results clearly demonstrate that the pristine PLGA has nonbactericidal nature. On the other hand, the PLGA/CuO hybrid nanofiber scaffold produced zones of inhibition against both $E$. coli and S. aureus (Figure 5(a)) indicating antibacterial activity based on the incorporation of $\mathrm{CuO}$ into PLGA nanofiber. Thus PLGA/CuO hybrid nanofiber scaffold has obtained the bactericidal ability. A similar phenomenon was also indicated by the optical density method (Figure 5(b)). An increasing trend in the cell density is observed in the samples containing PLGA; however, the growth of both the E. coli and $S$. aureus is greatly inhibited by PLGA/CuO nanofiber scaffold. The inhibition trend remained the same as was shown by disc diffusion method; that is, E. coli is inhibited more as compared to $S$. aureus. Thus it is concluded that the disc diffusion as well as optical density method showed significant (Figures 5(a) and 5(b)) antibacterial activity of PLGA/CuO nanofiber scaffold. The antibacterial activities of $\mathrm{Cu}$ and $\mathrm{CuO}$ are well established [24]. $\mathrm{CuO}$, not only in colloidal form but also in composite form, have also shown tremendous antibacterial properties [20, 25]. Herein the produced inhibition zones and the higher inhibition in the growth of both $E$. coli and $S$. aureus could be attributed to the bactericidal nature of $\mathrm{CuO}$ [26-28].

Schematics of the potential mechanisms which can be effective in retarding the bacterial growth are depicted in Figure 6. Exact mechanism of the antibacterial activities of nanomaterials is still not known $[2,29]$. The bacterial growth can be inhibited by the penetration of $\mathrm{CuO}$ nanoparticles or $\mathrm{Cu}^{++}$ions into the cell via cell membrane or may inhibit bacterial growth by the reactive oxygen species (ROS) generation. However, the most established mechanism is the adhesion of $\mathrm{CuO}$ nanoparticles $/ \mathrm{Cu}^{++}$ions to the protein containing sulphur present in the cell wall of the bacterial 


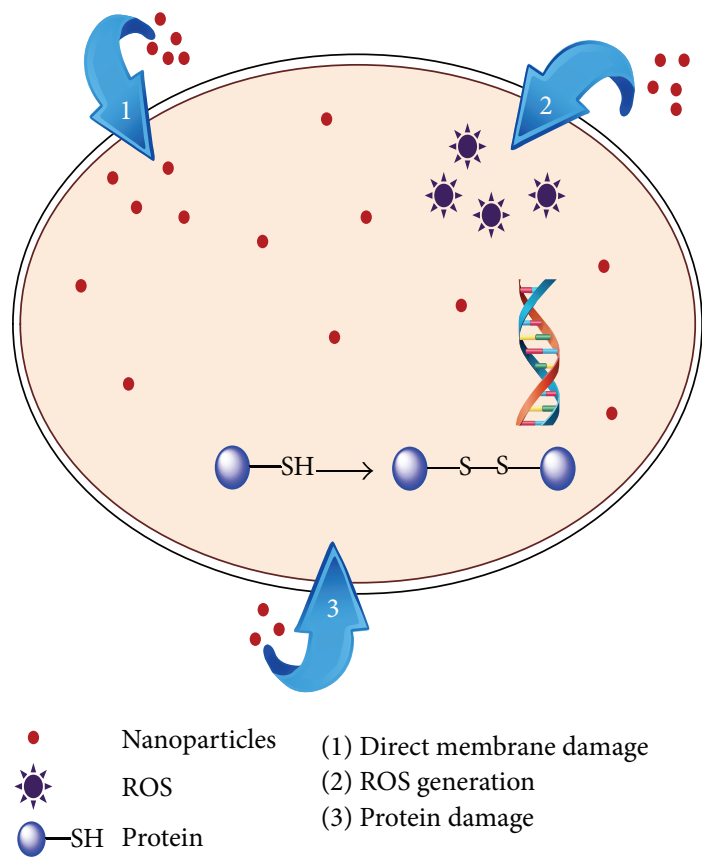

FIGURE 6: Schematic representing mechanism related to the antibacterial activity of $\mathrm{CuO}$ nanoparticles/ $\mathrm{Cu}^{++}$ions.

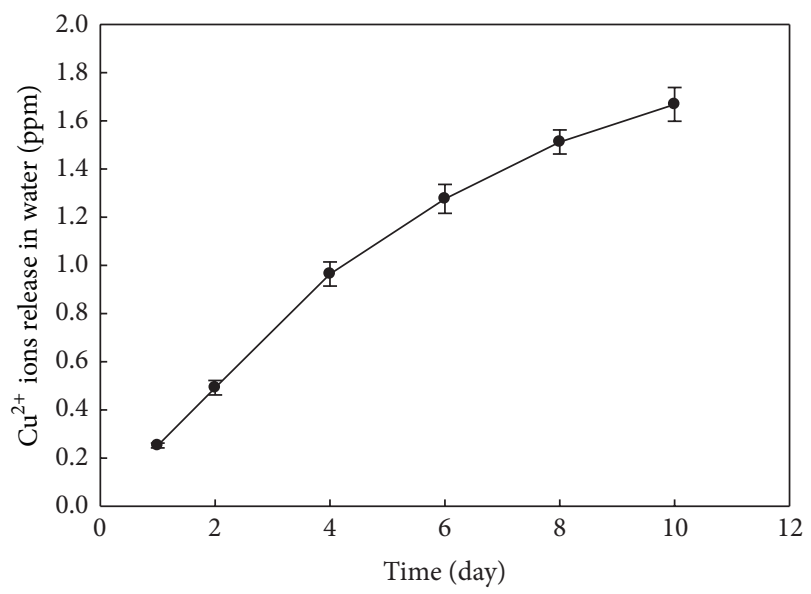

FIgURe 7: $\mathrm{Cu}^{++}$ions release in water from the PLGA/CuO hybrid nanofiber scaffolds with respect to incubation time.

cells, which leads to the malfunctioning of the bacterial cell wall and ultimately causes the bacterial cell death $[27,28,30]$.

3.4. $\mathrm{Cu}^{++}$Ions Release Study. Figure 7 depicts the results obtained from the $\mathrm{Cu}^{++}$ions release experiment. It was observed that $\mathrm{Cu}^{++}$ions from the PLGA/CuO hybrid nanofiber scaffold were released in a sustained manner, therefore enabling the PLGA/CuO hybrid nanofiber scaffolds to exhibit antibacterial effect for longer duration. The amount of $\mathrm{Cu}^{++}$ions released from the PLGA/CuO hybrid nanofiber scaffold reached $1.6 \mathrm{ppm}$ after 10 days of incubation in water. The $\mathrm{Cu}^{++}$ions release and the inhibition of $E$. coli and $S$. aureus by the PLGA/CuO nanofiber scaffold (Figure 5(b)) follow the same pattern; this is an indication that the operating mechanism of inhibition is governed by $\mathrm{Cu}^{++}$ ions. However, for $\mathrm{Cu}^{++}$ions release, the choice of media is important, as the body fluid has a complex composition and the components comprising body fluid exhibit different binding abilities to $\mathrm{Cu}^{++}$ions $[28,31,32]$. The amount of $\mathrm{Cu}^{++}$ions in the double distilled water was determined by inductively coupled plasma spectrophotometer.

\subsection{Cytocompatibility Study}

3.5.1. Cell Adhesion. NIH3T3 cells tend to get adhered to the surface of the biocompatible materials; therefore the various cell fate processes including proliferation, migration, apoptosis, and differentiation are highly affected by cells adhesion to cell-binding epitopes in the extracellular matrix (ECM) [33]. Figure 8 depicts the FE-SEM images of the NIH3T3 cells adhered to the pristine PLGA and PLGA/CuO hybrid nanofiber scaffolds with different incubation time. It is clearly visible that NIH3T3 cells were adhered to both pristine PLGA (Figures 8(a) and 8(c)) and PLGA/CuO hybrid nanofiber scaffolds (Figures $8(\mathrm{~b})$ and $8(\mathrm{~d})$ ), thus proving that both pristine PLGA and PLGA/CuO hybrid nanofiber scaffolds provided good cytocompatibility environment for NIH3T3 cells. The spreading pattern of the NIH3T3 cells on the PLGA and PLGA/CuO hybrid nanofiber scaffolds further confirmed the noncytotoxic nature of the scaffolds. Furthermore, the observed increase in the number of adhered cells to the scaffolds was directly proportional to the increase in incubation time (Figures 8(c) and 8(d)) $[3,12]$.

3.5.2. Cell Proliferation and Viability. Many colorimetric methods have been adapted in order to estimate the exact cell number, among which the methods based on cells metabolic viability test are widely employed [34]. MTT reagents are widely used for this purpose. Figure 9 illustrates the data obtained from MTT assay of the proliferation of fibroblastic cells seeded on the PLGA and PLGA/CuO hybrid nanofiber scaffolds. From the MTT assay it was observed that NIH3T3 cells proliferated on the PLGA and PLGA/CuO hybrid nanofiber scaffolds. The NIH3T3 cells proliferation on the PLGA/CuO hybrid nanofiber scaffolds was in the range of standard deviation compared with pristine PLGA nanofiber; thus both scaffolds exhibit cytocompatibility nature. Furthermore, MTT assay was conclusive in elucidating the proliferation of NIH3T3 cells on the pristine PLGA and PLGA/CuO hybrid nanofiber scaffolds.

Figures 10(a) and 10(b) show the fluorescence images of the NIH3T3 cells cultured on the PLGA/CuO hybrid nanofiber scaffold for 8 days using the live/dead assay [23]. The fluorescence color of cells cultured on the PLGA and PLGA/CuO hybrid nanofiber scaffolds was totally green, indicating a good viability of the fibroblastic cells. Rarely, few dead cells showed red fluorescence of propidium iodide staining (Figure 10(b)) when cultured on the PLGA/CuO hybrid nanofiber scaffolds [3]. These observations of the live/dead assay clearly suggest that the PLGA/CuO nanofiber 


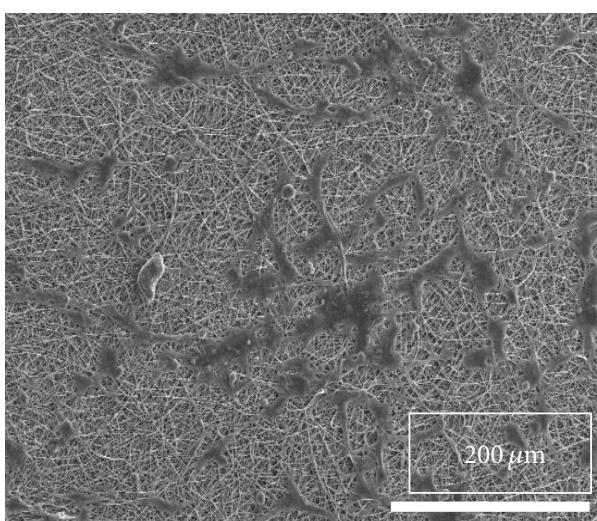

(a)

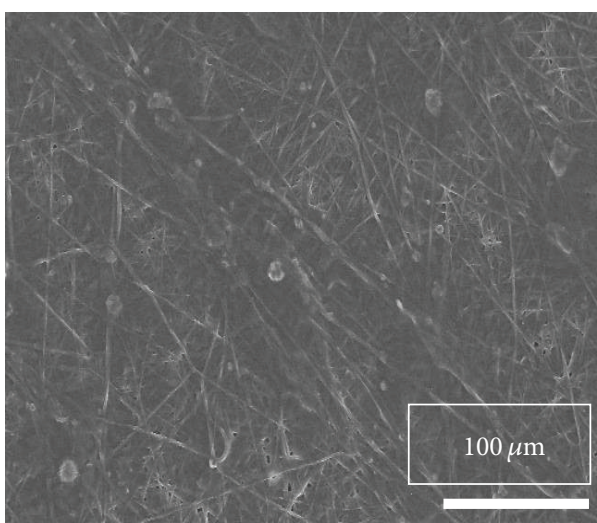

(c)

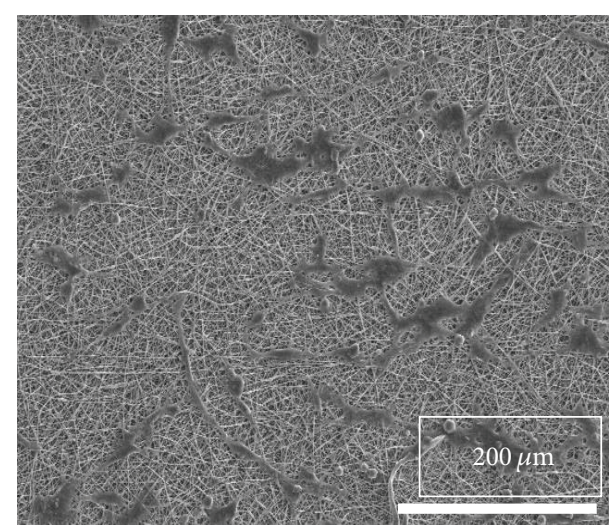

(b)

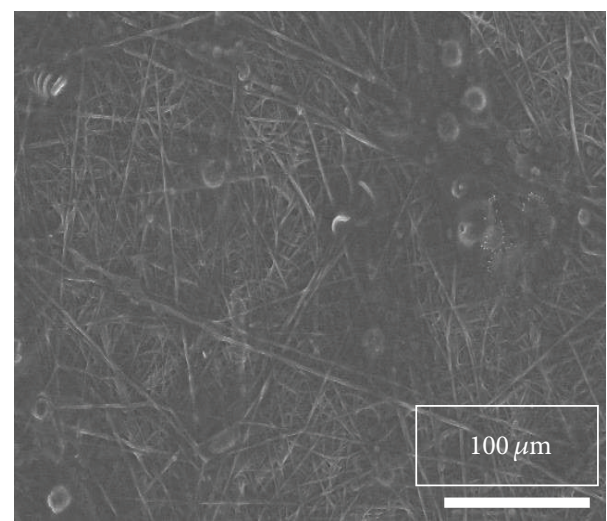

(d)

FIGURE 8: FE-SEM images of NIH3T3 cells adhered to the pristine PLGA (a, c) and PLGA/CuO hybrid nanofiber scaffolds (b, d) after 1 and 3 days of incubation.

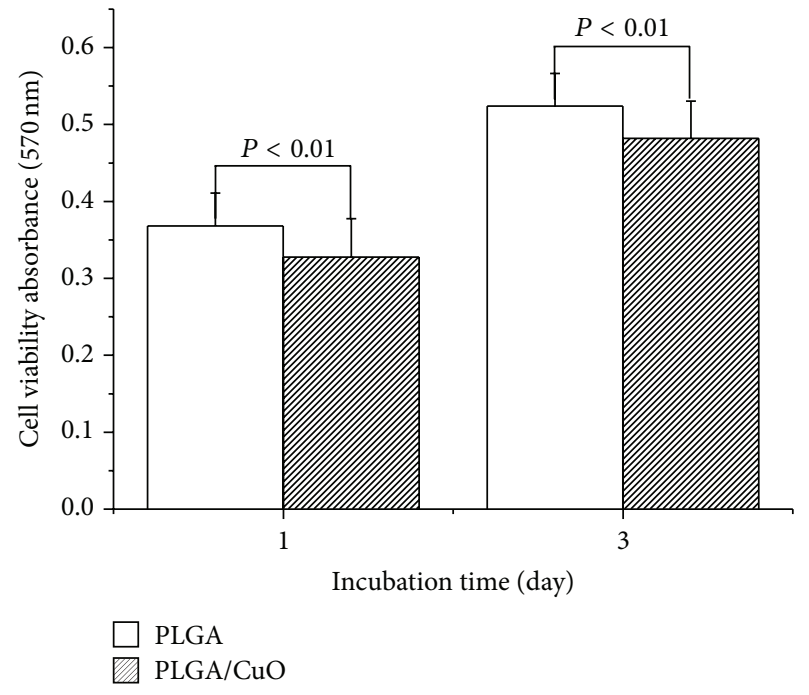

FIGURE 9: MTT assay after culturing of fibroblastic cells on the pristine PLGA and PLGA/CuO after 1 and 3 days of incubation.

scaffolds provided favorable environment to NIH3T3 cells [3].

\section{Conclusion}

This study was designed to evaluate the antibacterial activity along with assessing the biocompatibility of the pristine PLGA and PLGA/CuO hybrid nanofiber scaffolds. Prior to the evaluation of the antimicrobial activity, PLGA and PLGA/CuO hybrid nanofiber scaffolds were characterized using FE-SEM, TEM, and XPS. PLGA and PLGA/CuO nanofiber scaffolds showed excellent antibacterial activity against $E$. coli and $S$. aureus bacterial strains. The mechanism of the antibacterial activity was concluded to be based on the $\mathrm{Cu}^{++}$ion release. Furthermore, the various tests performed for the assessment of cytocompatibility using NIH3T3 cells showed good cytocompatibility for both PLGA and PLGA/CuO nanofiber scaffolds. It is therefore concluded from the antibacterial tests and in vitro cell experiments that PLGA/CuO nanofiber scaffolds have the potential to be used as a wound dressing material.

\section{Conflict of Interests}

The authors have no conflict of interests regarding the publication of this paper. 


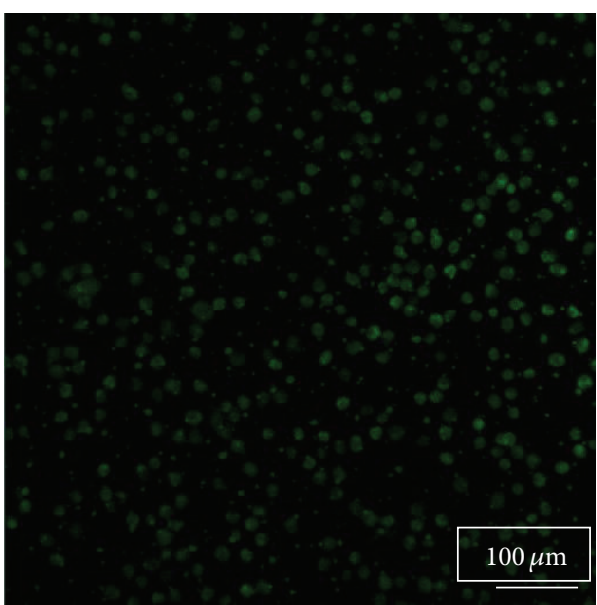

(a)

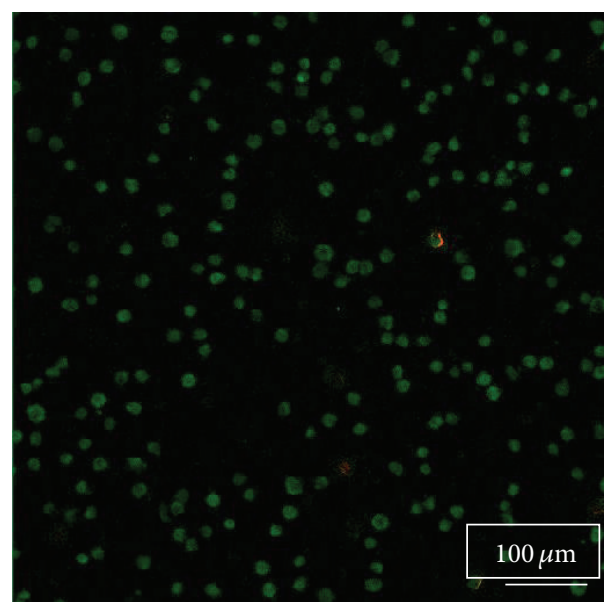

(b)

FIGURE 10: Fluorescence images of NIH3T3 cells cultured on pristine PLGA (a) and PLGA/CuO (b) fibroblastic cells after 3 days of culture.

\section{Acknowledgments}

This work was supported by the General Research Program (2013 RIAIA 2005148) and the Basic Research Laboratory program (no. 2011-0020264) from the Ministry of Education, Science and Technology of Korea.

\section{References}

[1] C. Marambio-Jones and E. M. V. Hoek, "A review of the antibacterial effects of silver nanomaterials and potential implications for human health and the environment," Journal of Nanoparticle Research, vol. 12, no. 5, pp. 1531-1551, 2010.

[2] K. Chaloupka, Y. Malam, and A. M. Seifalian, "Nanosilver as a new generation of nanoproduct in biomedical applications," Trends in Biotechnology, vol. 28, no. 11, pp. 580-588, 2010.

[3] Z.-C. Xing, W.-P. Chae, J.-Y. Baek, M.-J. Choi, Y. Jung, and I.$\mathrm{K}$. Kang, "In vitro assessment of antibacterial activity and cytocompatibility of silver-containing phbv nanofibrous scaffolds for tissue engineering," Biomacromolecules, vol. 11, no. 5, pp. 1248-1253, 2010.

[4] M. Rai, A. Yadav, and A. Gade, "Silver nanoparticles as a new generation of antimicrobials," Biotechnology Advances, vol. 27, no. 1, pp. 76-83, 2009.

[5] P. M. Narayanan, W. S. Wilson, A. T. Abraham, and M. Sevanan, "Synthesis, characterization, and antimicrobial activity of zinc oxide nanoparticles against human pathogens," BioNanoScience, vol. 2, no. 4, pp. 329-335, 2012.

[6] M. Ul-Islam, A. Shehzad, S. Khan, W. A. Khattak, M. W. Ullah, and J. K. Park, "Antimicrobial and biocompatible properties of nanomaterials," Journal of Nanoscience and Nanotechnology, vol. 14, pp. 1-12, 2014.

[7] N. Cioffi, L. Torsi, N. Ditaranto et al., "Copper nanoparticle/polymer composites with antifungal and bacteriostatic properties," Chemistry of Materials, vol. 17, no. 21, pp. 5255-5262, 2005.

[8] M. Ahamed, H. A. Alhadlaq, M. A. M. Khan, P. Karuppiah, and N. A. Al-Dhabi, "Synthesis, characterization, and antimicrobial activity of copper oxide nanoparticles," Journal of Nanomaterials, vol. 2014, Article ID 637858, 4 pages, 2014.
[9] G. Ren, D. Hu, E. W. C. Cheng, M. A. Vargas-Reus, P. Reip, and R. P. Allaker, "Characterisation of copper oxide nanoparticles for antimicrobial applications," International Journal of Antimicrobial Agents, vol. 33, no. 6, pp. 587-590, 2009.

[10] Y. Zhang, N. Zheng, K. Wang, S. Zhang, and J. Wu, "Effect of copper nanoparticles dispersion on catalytic performance of $\mathrm{Cu} / \mathrm{SiO}_{2}$ catalyst for hydrogenation of dimethyl oxalate to ethylene glycol," Journal of Nanomaterials, vol. 2013, Article ID 629375, 6 pages, 2013.

[11] K. A. Khalil, H. Fouad, T. Elsarnagawy, and F. N. Almajhdi, "Preparation and characterization of electrospun PLGA/silver composite nanofibers for biomedical applications," International Journal of Electrochemical Science, vol. 8, no. 3, pp. 34833493, 2013.

[12] A. Haider, K. Gupta, and I.-K. Kang, "PLGA/nHA hybrid nanofiber scaffold as a nanocargo carrier of insulin for accelerating bone tissue regeneration," Nanoscale Research Letters, vol. 9, no. 1, pp. 314-326, 2014.

[13] A. Haider, K. C. Gupta, and I.-K. Kang, "Morphological effects of HA on the cell compatibility of electrospun HA/PLGA composite nanofiber scaffolds," BioMed Research International, vol. 2014, Article ID 308306, 11 pages, 2014.

[14] S. Haider, Y. Al-Zeghayer, F. A. A. Ali et al., "Highly aligned narrow diameter chitosan electrospun nanofibers," Journal of Polymer Research, vol. 20, no. 4, article 105, 2013.

[15] S. Haider, W. A. Al-Masry, N. Bukhari, and M. Javid, "Preparation of the chitosan containing nanofibers by electrospinning chitosan-gelatin complexes," Polymer Engineering \& Science, vol. 50, no. 9, pp. 1887-1893, 2010.

[16] H.-S. Bae, A. Haider, K. M. K. Selim, D.-Y. Kang, E.-J. Kim, and I.-K. Kang, "Fabrication of highly porous PMMA electrospun fibers and their application in the removal of phenol and iodine," Journal of Polymer Research, vol. 20, no. 7, article 158, 7 pages, 2013.

[17] V. Pillay, C. Dott, Y. E. Choonara et al., "A review of the effect of processing variables on the fabrication of electrospun nanofibers for drug delivery applications," Journal of Nanomaterials, vol. 2013, Article ID 789289, 22 pages, 2013.

[18] N. Bhardwaj and S. C. Kundu, "Electrospinning: a fascinating fiber fabrication technique," Biotechnology Advances, vol. 28, no. 3, pp. 325-347, 2010. 
[19] M. A. Shah and M. S. Al-Ghamdi, "Preparation of copper (Cu) and copper oxide $\left(\mathrm{Cu}_{2} \mathrm{O}\right)$ nanoparticles under supercritical conditions," Materials Sciences and Application, vol. 2, no. 8, pp. 977-980, 2011.

[20] M. Ul-Islam, T. Khan, W. A. Khattak, and J. K. Park, "Bacterial cellulose-MMTs nanoreinforced composite films: novel wound dressing material with antibacterial properties," Cellulose, vol. 20, no. 2, pp. 589-596, 2013.

[21] T. Maneerung, S. Tokura, and R. Rujiravanit, "Impregnation of silver nanoparticles into bacterial cellulose for antimicrobial wound dressing," Carbohydrate Polymers, vol. 72, no. 1, pp. 4351, 2008.

[22] S. A. Holowachuk, M. F. Bal'a, and R. K. Buddington, "A kinetic microplate method for quantifying the antibacterial properties of biological fluids," Journal of Microbiological Methods, vol. 55, no. 2, pp. 441-446, 2003.

[23] K. Trescher, N. Scharnagl, K. Kratz, T. Roch, A. Lendlein, and F. Jung, "Adherence and viability of primary human keratinocytes and primary human dermal fibroblasts on acrylonitrile-based copolymers with different concentrations of positively charged functional groups," Clinical Hemorheology and Microcirculation, vol. 52, no. 2-4, pp. 391-401, 2012.

[24] V. K. Champagne and D. J. Helfritch, "A demonstration of the antimicrobial effectiveness of various copper surfaces," Journal of Biological Engineering, vol. 7, article 8, 2013.

[25] Y. Haldorai and J.-J. Shim, "Multifunctional chitosan-copper oxide hybrid material: photocatalytic and antibacterial activities," International Journal of Photoenergy, vol. 2013, Article ID 245646, 8 pages, 2013.

[26] A. A. Tayel, W. F. El-Tras, S. Moussa et al., "Antibacterial action of zinc oxide nanoparticles against foodborne pathogens," Journal of Food Safety, vol. 31, no. 2, pp. 211-218, 2011.

[27] A. Azam, A. S. Ahmed, M. Oves, M. S. Khan, S. S. Habib, and A. Memic, "Antimicrobial activity of metal oxide nanoparticles against Gram-positive and Gram-negative bacteria: a comparative study," International Journal of Nanomedicine, vol. 7, pp. 6003-6009, 2012.

[28] X. Hu, S. Cook, P. Wang, and H.-M. Hwang, "In vitro evaluation of cytotoxicity of engineered metal oxide nanoparticles," Science of The Total Environment, vol. 407, no. 8, pp. 3070-3072, 2009.

[29] B. Reidy, A. Haase, A. Luch, K. A. Dawson, and I. Lynch, "Mechanisms of silver nanoparticle release, transformation and toxicity: a critical review of current knowledge and recommendations for future studies and applications," Materials, vol. 6, no. 6, pp. 2295-2350, 2013.

[30] A. Haider and I.-K. Kang, "Preparation of silver nanoparticles and their industrial and biomedical applications: a comprehensive review," Advances in Materials Science and Engineering, vol. 2014, Article ID 165257, 16 pages, 2014.

[31] A. Semisch, J. Ohle, B. Witt, and A. Hartwig, "Cytotoxicity and genotoxicity of nano- and microparticulate copper oxide: role of solubility and intracellular bioavailability," Particle and Fibre Toxicology, vol. 11, article 10, 2014.

[32] M. S. Hassan, T. Amna, H. Y. Kim, and M.-S. Khil, "Enhanced bactericidal effect of novel $\mathrm{CuO} / \mathrm{TiO}_{2}$ composite nanorods and a mechanism thereof," Composites Part B: Engineering, vol. 45, no. 1, pp. 904-910, 2013.

[33] X. Cao, K. Kwek, J. K. Y. Chan, C. K. H. Chan, and M. Lim, "Electrospun nanofibers as a bioadhesive platform for capturing adherent leukemia cells," Journal of Biomedical Materials Research Part A, vol. 102, no. 2, pp. 523-531, 2014.
[34] A. G. Karakecili, T. T. Demirtas, C. Satriano, M. Gümüsderelioglu, and G. Marletta, "Evaluation of L929 fibroblast attachment and proliferation on Arg-Gly-Asp-Ser (RGDS)immobilized chitosan in serum-containing/serum-free cultures," Journal of Bioscience and Bioengineering, vol. 104, no. 1, pp. 69-77, 2007. 

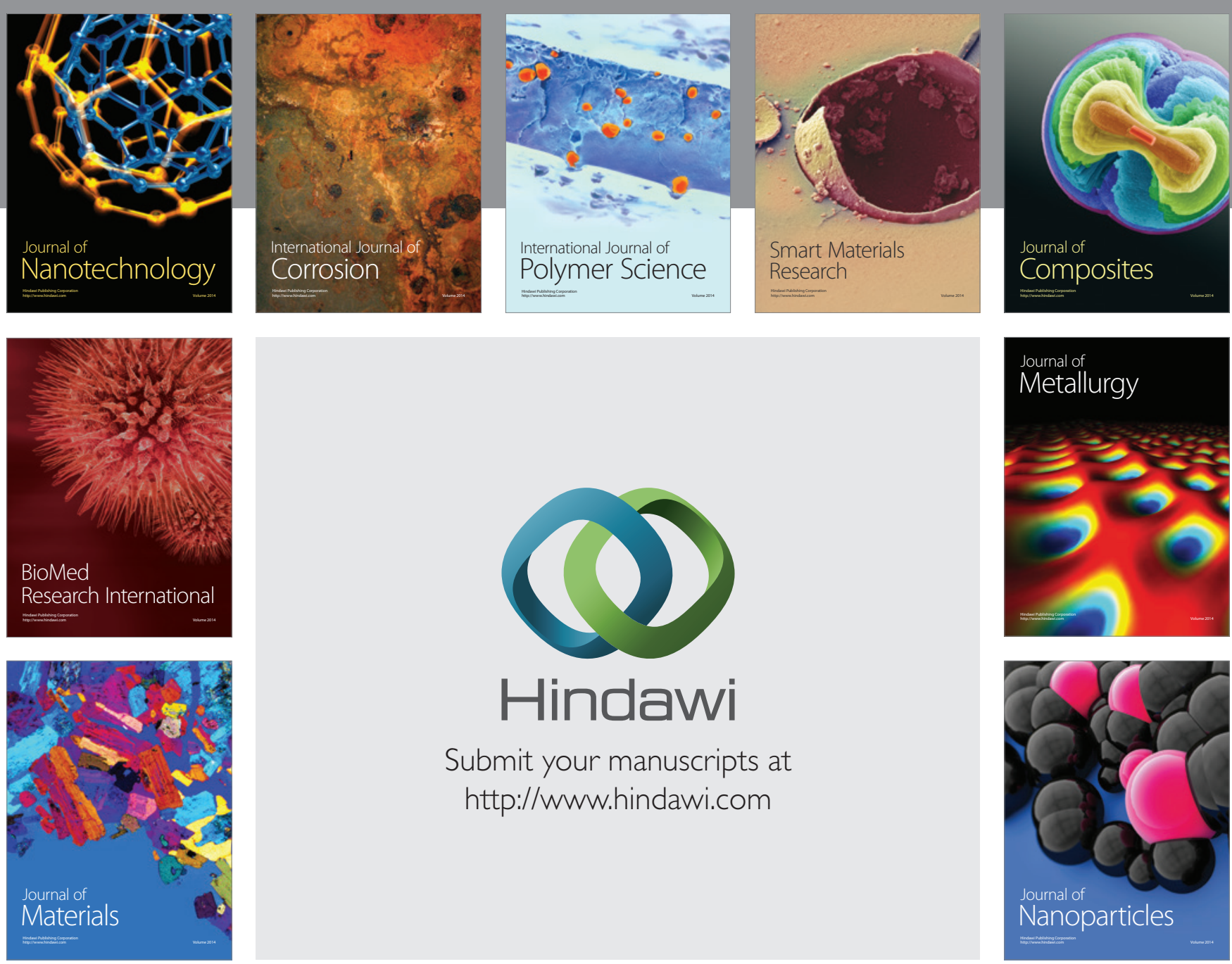

Submit your manuscripts at http://www.hindawi.com
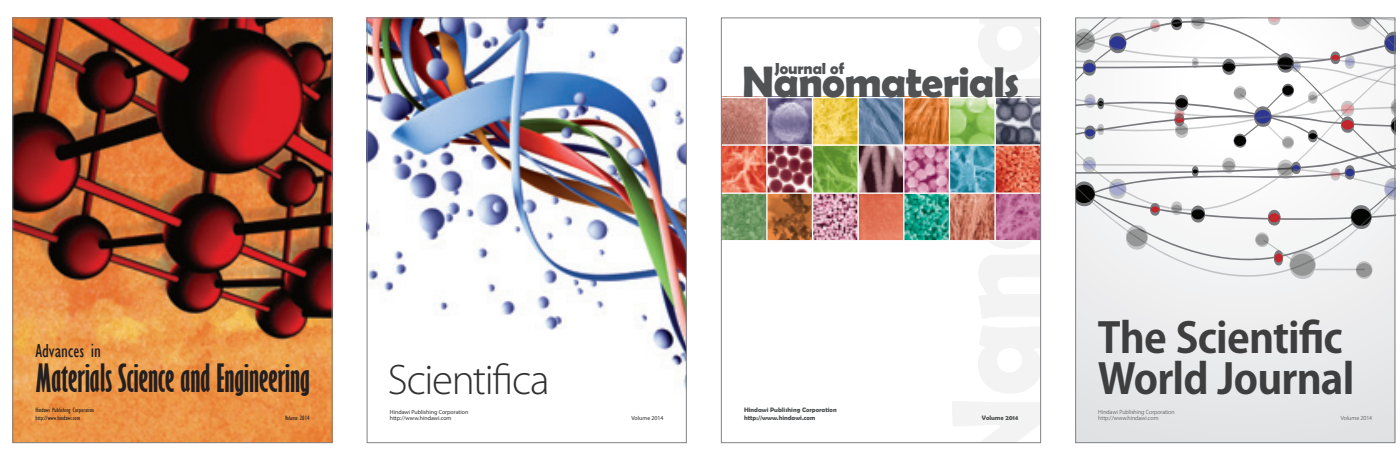

\section{The Scientific World Journal}
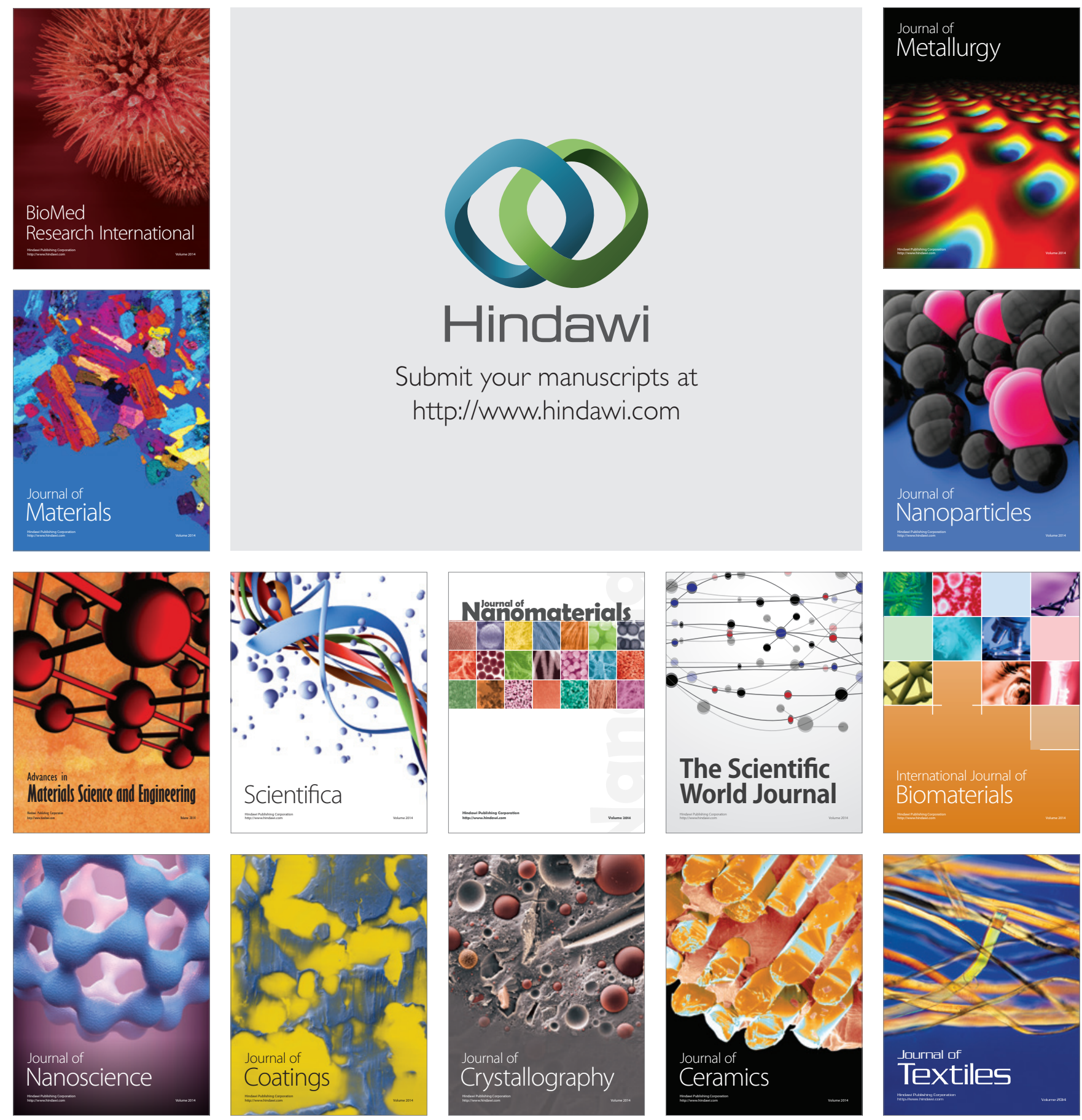